\title{
Advanced level preparation of Expanded Graphite, lauric acid, Stearic acid for heat transfer and solar applications
}

1. ANUTSEK SHARMA

2. DR. S. KARTHIKEYAN

3. RATCHAGAR PRABHU

4. DR.KARTHIKEYAN JAGANNATHAN

1 CAU KIEL,GERMANY

2,3,4 SRM UNIVERSITY, CHENNAI, INDIA

\begin{abstract}
Today with enhancement in technology, sciences, there is also an increase in global heating rate. There is an urgent need of any alternate efficient source to reduce the wastage of energy and to utilize it efficiently. The advanced preparation of Expanded graphite ,lauric acid, stearic acid as shape stabilized phase change material deals with different energy harvesting applications. The main reason behind the need for synthesis of this matrix is to prepare a material that can be used in low temperature energy storage applications. Mixture of lauric acid, stearic acid impregnated in expanded graphite through vacuum impregnation followed by Vacuum Drying and Microwave acid treatment serves as novel shape stabilized phase change material of its type. The microwave acid treatment was done in order to increase the removal of moisture from the sample thus initiating proper bonding of its constituents. The mixture was produced in 1:1:1 ratio where all expanded graphite, lauric acid, stearic acid has one proportions of each other. The product obtained after microwave acid treatment was subjected to SEM, DSC analysis.
\end{abstract}

\section{KEYWORDS}

SSPCM, Vacuum Drying, Microwave Acid Treatment, Solar Applications

\section{INTRODUCTION}

The phase change materials (PCM) are a type of latent heat storage material. They deliver heat at constant rate. The thermal heat transfer occurs as a result of solidyfing and melting, which is the reason these matrials are called as phase change materials. The shape stabilized phase change material (SSPCM) is an important class of phase change materials. These materials donot require an extra encapsulaton which was the case with PCM. In SSPCM shape of the material is stabilized while the heal transfer occurs in similar way as in PCM. Offen this is observed that PCM looses its properties at intermediate temperature or high temprature. The aim of this paper is to develop SSPCM which can withstand the temeprature of atleast $90^{\circ} \mathrm{C}$. However the application of the produced product is not yet implemented but with the help of DSC, it is proved that the sample can withstand temperature near to $92^{\circ} \mathrm{C}$ approximately. Many researcher have worked on the heat transfer efficiency enhancing procedure like large fins, where Nagano et al used micro encapsulated PCM mixture and salt hydrate as energy harvesting matrial (1). However, it was observed that suffcicent increase in number of fins doesnot enhance the heat transfer. Tayeb et al used metal slags and chips to 
enhance the effective thermal conductivity (2). However, Satzger and Eska et al proposed an idea of harvsting heat in a PCM embedded matrix of expanded graphite (3). Fukai et al studied heat transfering techiniqe with the use of carbon fibers, these cabon fibers have heat condctivity close to auminium and copper (4). PCM has found its application in wide range of inapplications like Solar water heating systems, Solar air heating systems, Solar cookers, Solar green house, PCM trombe wall etc (5). There are different types of synhesis process available for PCM, out of which the most common ones are vacuum impregnation and etahnol wash. The experimental processes opted for this work will be discussed in next section.

\section{MATERIAL SELECTION AND EXPERIMENT}

Before starting the experiment to synthesize shape stabilized phase change materials, standard procedure was opted and was performed in proper guidance. The real time procedure included selection of material. This was started by selecting a supporting material and a phase change material. Selection criteria were considered before selecting the supporting material and phase change material like property, heat exchange rate, thermal coefficient, availability, cost, melting point, boiling point and so on. Finally Expanded Graphite is used as a supporting material because it can be produced in laboratory easily and was having high heat exchanging rate. Lauric acid, stearic acid were chosen as the phase change material due to their low melting point, low boiling point and its property of good thermal co- efficiency. After this process, vacuum impregnation was performed and this would become more clear with Figure1.

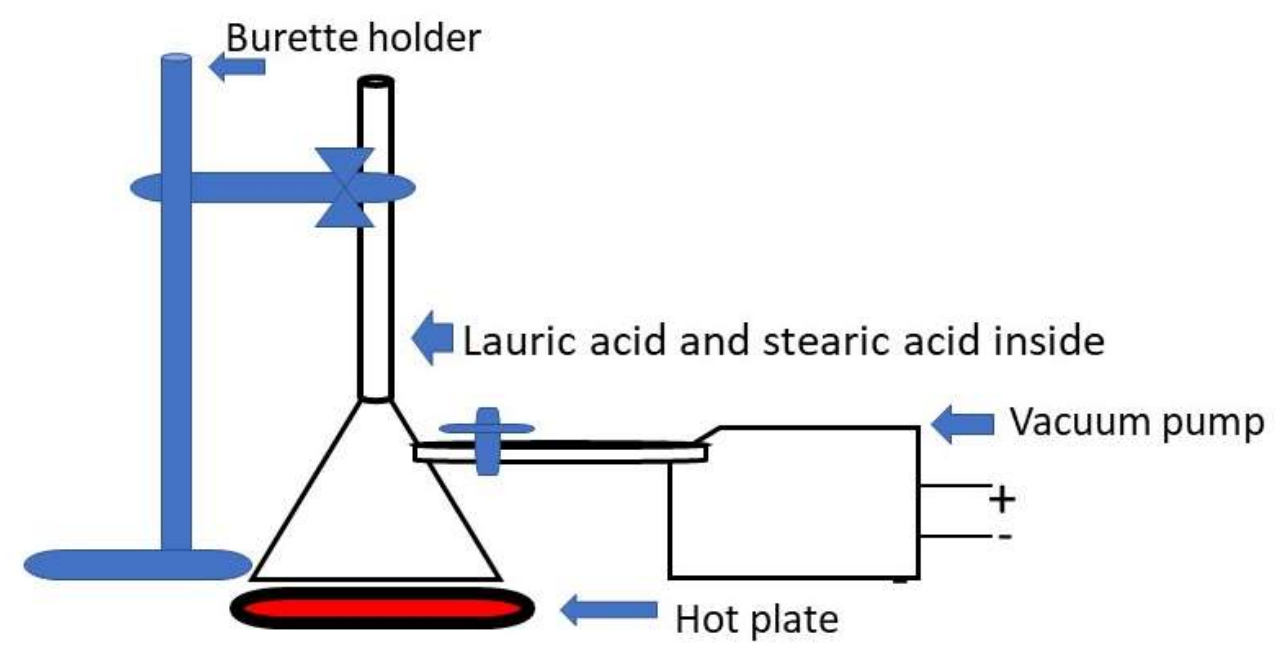

Figure 1 - Setup for Vacuum Impregnation

Lauric acid, stearic acid was chosen as the phase change material which was used in the process of vacuum impregnation. In this paragraph, a detailed 
description of how vacuum impregnation was performed will be discussed. This experiment was done in the ratio of 1:1:1 where one part of Expanded Graphite was used and one -one parts of Lauric acid - stearic acid solution was used. Thus, during vacuum impregnation technique, vacuum was created for almost 2-3 hours till the pressure drop was almost 2-3 $\mathrm{Hg}$ on pressure gauge. Apart from this, vacuum pump was used to perform vacuum impregnation operation. This invariably gave the rough outlook of how the operation should be carried out. Moreover, a pressure valve was involved in this process to control the amount of pressure inside the filtration flask. The hot plate acted as the major component in removing the moisture from the flask. Before performing vacuum impregnation, the flask was heated for almost one hour at 70-90 degree centigrade. A rubber cork and burette was also involved in this process. The process starts by formation of a Lauric acid-Stearic acid solution by mixing powder Lauric acid-Stearic acid with almost 30-45 $\mathrm{ml}$ of water and stirring it on magnetic stirring equipment for 2 hours at a temperature of 70 degrees. When the above described process gets over, a visible white solution of Lauric acid appears in the beaker. Filtration flask is filled with Expanded Graphite (the supporting material, in this case) on which the vacuum impregnation was performed. During this process, the formation of vacuum is done by connecting filtration flask, pressure valve, pressure gauge and vacuum pump by a pipe of required diameter. The vacuum pump is of 150 watt capacity, which is operated at a regular interval of 5-10 minutes. This was performed for 3 hours till the required pressure was achieved. When the setup was properly arranged, the burette was filled with the solution of Lauric acid- Stearic acid powder and water. From this time, impregnation of Lauric acidStearic acid solution on Expanded Graphite was done with vacuum that was created with the help of the pump. During the impregnation, a lot of smoke and foul odour was produced (due to heating) which later was given out by the vacuum pump. This process took 2 hours approx. After this, the sample obtained was vacuum dried for amost 2 hours. This was performed on hot plate and temperature was maintained at 80-90 degrees. This vigorous heating resulted in removal of moisture after some time. This process was then followed by micro wave acid treatment of 120 seconds at 750 watt. In this process the remaining amount of moisture from the sample would be removed.

After that crushing mixture was done to obtain fine particles and was later given for testing of SEM, and DSC.

\section{TESTING AND RESULTS}

- SEM ANALYSIS OF EXPANDED GRAPHITE 

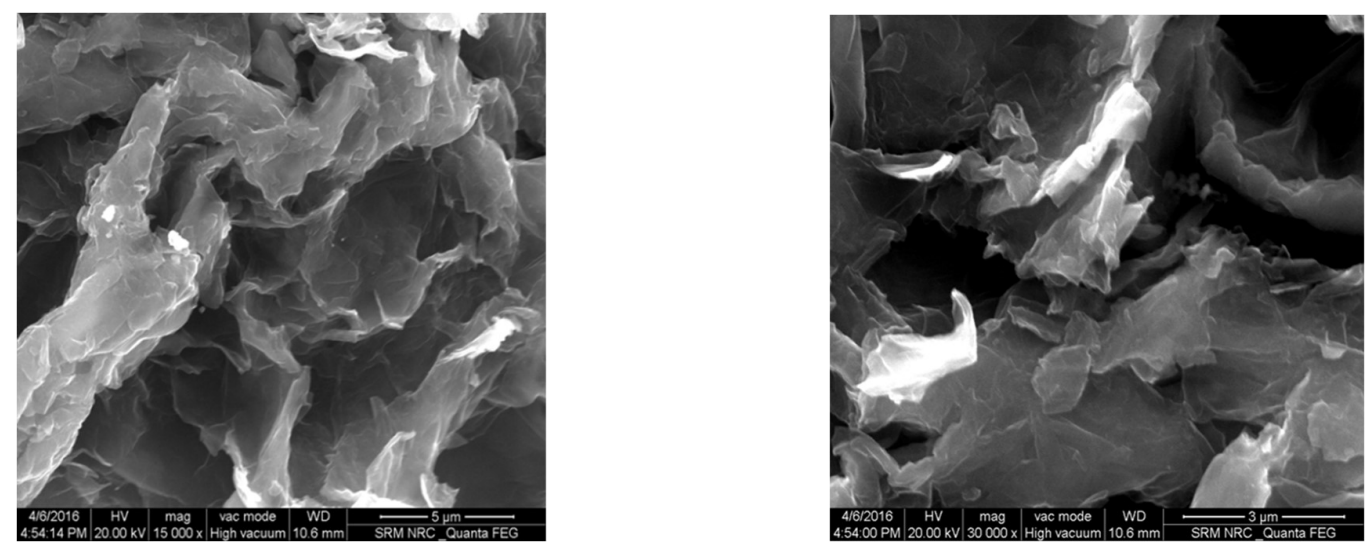

Figure 2 - FE-SEM analysis of Expanded Graphite at different magnifications; Low mangnification (Left image), High Magnification (Right image)

Figure 2 explains the FE-SEM analysis of Expanded Graphite done at IIT, MADRAS. The FE-SEM analysis explains the morphology of the material. Figure 2 shows the expanded graphite nano plates merged in each other forming a network. The most observable part here is the space between the nano plates of Expanded Graphite, this provides space for the Lauric acid and Stearic scid to get impregnated properly. The nano plates have non circular edges and due to this, the geometry of the powder is crystalline in nature.

\section{- SEM ANALYSIS OF POWDERED SAMPLE}

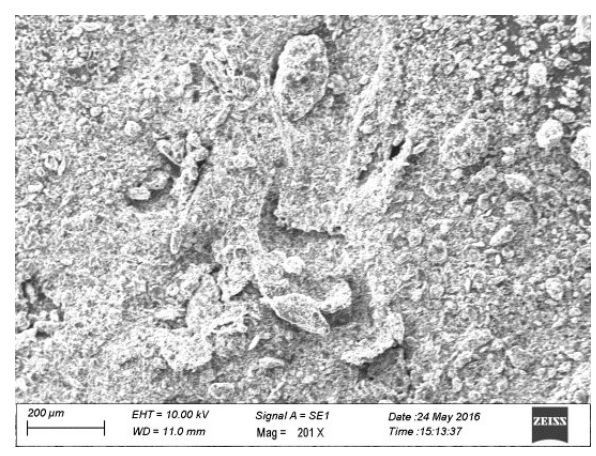

Figure 3 - SEM analysis of powdered sample of Expanded Graphite, Lauric acid and Stearic Acid.

Figure 3 explains the SEM analysis of powdered material with composition of Expanded Graphite, Lauric acid and Stearic Acid. The analysis was performed in ST. JOSEPHS COLLEGE, TRICHY. The SEM analysis explains the morphological analysis of component that is produced from Vacuum impregnation. The SEM analysis also shows the expanded graphite nano plates are adhered to the particles of lauric acid and stearic acid forming a rough surface structure with some regidity. The crystallinity of the sample is also reduced, when compared to FE-SEM analysis of Expanded graphite. The possible reason for this is, the liquid solution of Stearic and Lauric acid which has properly impregnated at the surface and in the spaces of expanded graphite powder thus forming a layered structure, affecting the crystallinity 
of the Expanded Graphite. Vacuum drying has reduced the amount of moisture from the sample which is also one of the reasons for the rough structure of the sample. This sem analysis was done before the microwave acid treatment.

- SEM ANALYSIS- AFTER ACID MICROWAVE TREATMENT
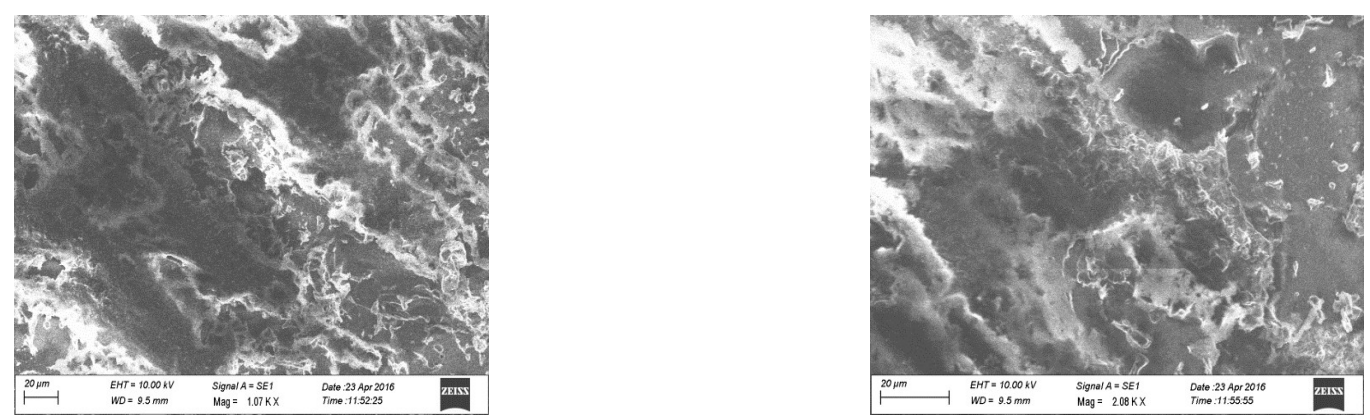

Figure 4 - SEM analysis after microwave treatment of the powdered sample, at different magnifications; Low mangnification (Left image), High Magnification (Right image)

Figure 4 explains the SEM analysis performed at ST. JOSEPHS COLLEGE, TRICHY. This analysis was done after the microwave acid treatment. The analysis has shown a highly integrated structure on exposure of powdered mixture in microwave acid treatment. The sample in powdered form was heated in a $750 \mathrm{watt}$ microwave at SRM University lab facility for $2 \mathrm{~min}$. Figure 4 also shows the solid structure and with very little to no crystallinity. The surface of the material appears to be intermideatly rough, this can be attributed to the fact that the remaining moisture had evaporated after microwave acid treatment. This evaporated moisture resulted in the proper bonding of Expanded graphite to Lauric acid and Stearic acid into the interestial spaces of Expanded Graphite flakes. The Lauric scid and Stearic acid have now properly bonded to Expanded graphite thus forming a flake like structure which can be seen in Figure 5

The below figure 5 shows the result of final product that we got after microwave acid treatment. It shows that the structure has lighweight, high integration, delicate and fused in each other. This Flake like material could be used in different heat transfer application and solar application.

The practicle use of this substance in the solar and other heat transfer applications, is beyond the scope of this paper. However, with the help of DSC testing of this sample it can be proved till what temperature the material can withstand its properties. 


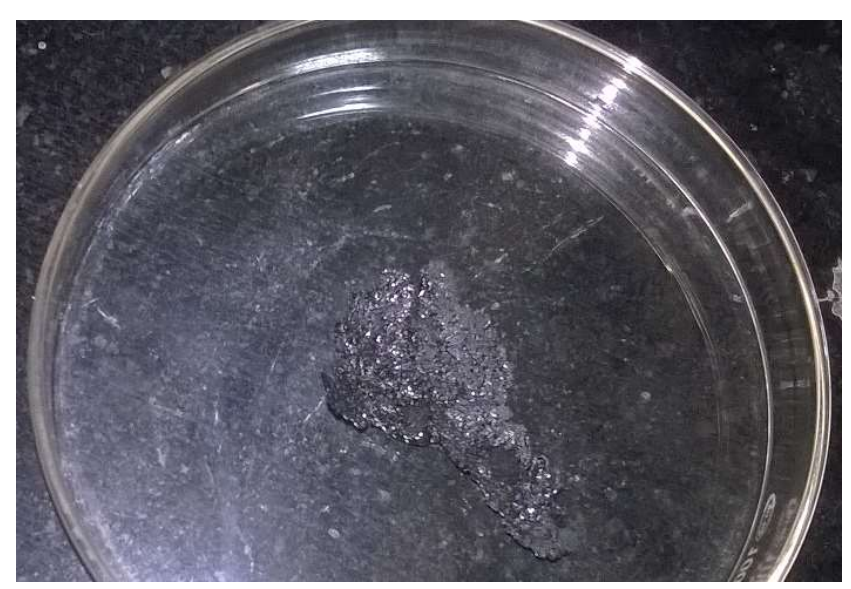

Figure 5 - Flake like structure of powdered Expanded Graphite, Lauric acid and Stearic acid after microwave acid treatment

- DSC TESTING OF POWDERED SAMPLE

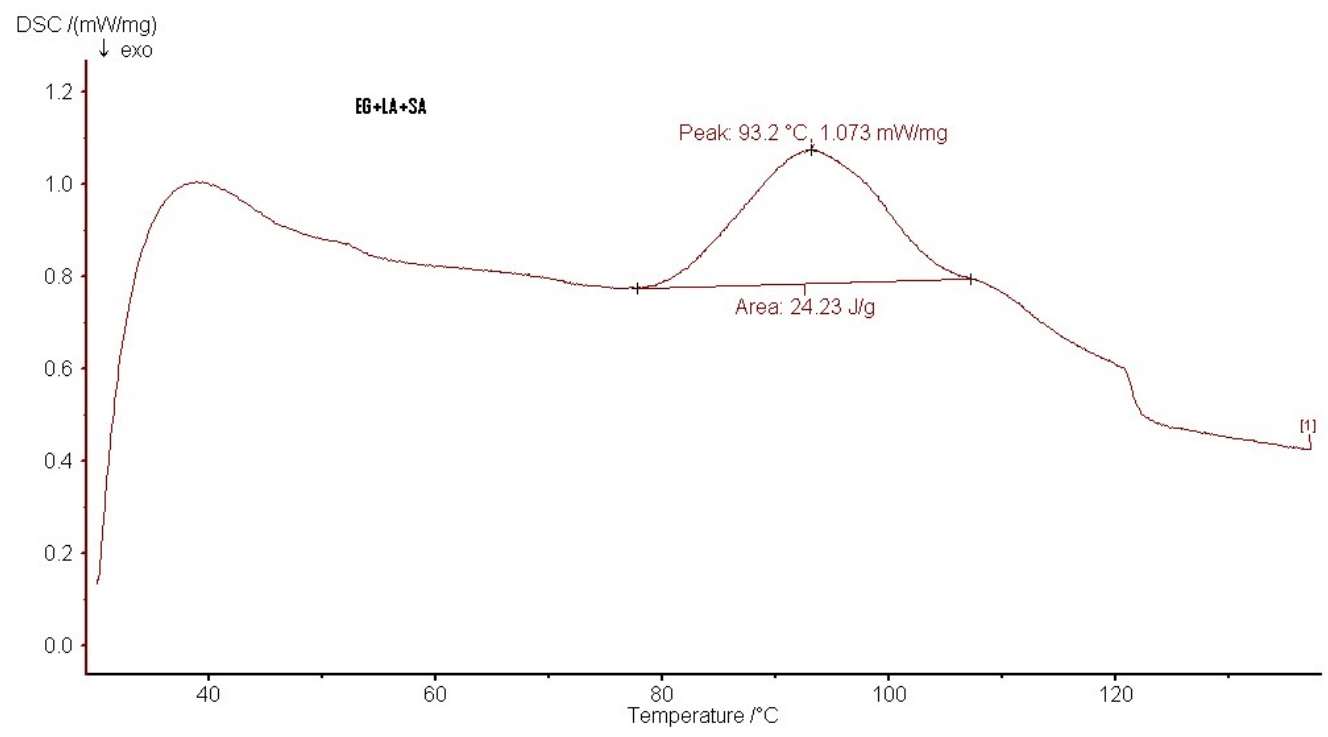

Figure 6 - DSC Testing of the powdered sample

Figure 6 explains the DSC Test that was performed in IIT ,MADRAS on the powdered sample of expanded graphite, steric acid, lauric acid which has gone through microwave acid treatment. The melting point of lauric acid is 40 degree and melting point of stearic acid is 60 degree (approximately). However, due to proper integration of the Lauric acid and Stearic acid into the Expanded Graphite, the matrix has resulted in increased melting point of the sample, i.e. $93.2^{\circ} \mathrm{C}$. From this result this is clear that the material produced can be used in various heat transfer application, this heat transfer application can be a application in utilizing solar energy. The material can withstand its properties till $93.2^{\circ} \mathrm{C}$. However, after $93.2^{\circ} \mathrm{C}$ the material properties may fail and it may stop responding to furthure heat transfer. The two times involved drying procedure namely, Vaccum Drying and Microwave Acid Treatment 
serves as an important steps of the fabrication process, the removal of maximum amount of moisture can be one of the reason for increased amount of melting temperature obtained from the material i.e. $93.2^{\circ} \mathrm{C}$. This also proves that extra drying also leads to increased level of thermal properties that can be obtained in form of increased melting point.

\section{CONCLUSION}

The synthesis and analysis of shape stabilized phase change material performed was found to be a novel one with promising result. The impregnation of stearic acid and lauric acid was as per the ratio of 1:1 which was accurate in expanded graphite. Also the result shown by DSC tesing explains and widens the application of this material from its use in solar storage application till its application ranging in construction to ceramic industry. The Microwave acid treatment done in the end was found to be an accurate cause of its high thermal efficiency and increased melting point. Procedure like vacuum drying was one of the verymost important pre-step to remove the moisture content from the material before it went to Microwave acid treatment. The material has also enhanced thermal properties due to reduced or no moisture content. Material is found to be suiatble in solar applications and in the applications where the melting point is below $93.2^{\circ} \mathrm{C}$.

\section{REFERENCE}

1. Nagamo $\mathrm{K}$ et al. Experiments on fin-effects for increasing heat transfer coefficients during charging heat and heat release between PCMs and thermal medium. 2004.

2. Tayeb et al. Use of some industrial wastes as energy storage media. Energy Conver Manage 37. (1996). 127-33.

3. Satzger P, Eska B et al. Matrix-heat-exchanger for latent-heat cold-storage. 1997.

4. Fukai J. Thermal conductivity enhancement of energy storage media using carbon fibers. Energy conversion and management 41 (14). (2000). 1543-1556.

5. Sharma et al. thermal energy storage with phase change materials and applications, Renew. Sustain. Energy Rev.13 (2009) 318-345. 